\title{
College Mathematics Teaching Based on HPS Teaching
}

\author{
Qiang Tang
}

\author{
China West Normal University, Nanchong, 637002, China
}

\begin{abstract}
Key words: HPS teaching, College mathematics, Implementation strategies
\end{abstract}
\begin{abstract}
The new educational concept of HPS has greatly influenced international scientific educational reform in recent years and promoted the development and progress of college mathematics teaching. Currently, the society has greater and greater demands for talents of mathematical science. Therefore, it is the only way which must be passed to apply HPS education to college mathematics teaching. This paper conducts discussions in this aspect, discusses specific ways of implementation and expects to manifest educational functions of college mathematical science, maximize its practical significance and provide comprehensive high-quality talents to promote social progress and scientific development.
\end{abstract}

\section{Introduction}

College mathematics teaching is an important part of scientific education as well as its foundation. In-depth understanding of students and college teachers about the essence of mathematical science and their good spirits of innovation and mathematical science are significant for the future development of Chinese nation. Therefore, it is an important issue to apply HPS teaching to college mathematics teaching, which is worth the common concern of educational workers.

\section{HPS and HPS teaching}

HPS is a new educational concept integrating the history of science, scientific sociology and the philosophy of science. HPS teaching refers to the application of HPS relevant theoretical contents in the field of educational science which allows students to have in-depth understanding of the essence of science and brand new understanding of science in the environment of history, philosophy and sociology and improves the scientific literacy of students and the quality of scientific education.

\section{College mathematical teaching based on HPS teaching is the only way to its development}

\section{Result of era development}

The overall background of globalization of science and technology promotes the formation of HPS educational trend. The globalization of science and technology refers to the implementation of science and technology in the whole society, thus making the society more scientific and technological. As the training base of higher talents, colleges and universities will aim at imparting scientific and technological knowledge and training corresponding practical skills so as to meet the increasing demand of the society for scientific and technological talents. However, science and technology are not perfect. Only with constant development and improvement can they benefit human beings. Therefore, colleges and universities should not relax in the training of students' ability of scientific innovation, scientific spirit, various professional abilities and social responsibility view. Such quality-oriented education is very important for the development of college students. Such educational concept of HPS advances with the times and is worth wide application in college mathematics teaching reform so as to actually manifest scientific educational function of college mathematics and allow students to learn mathematical knowledge and meanwhile have profound and comprehensive understandings of social value and essence of mathematics and become mathematical experts with strong sense of social responsibility and innovation spirit. 


\section{Inevitable requirements of the combination between scientific education of college mathematics and humanistic spirit education}

With faster development of science and technology, people can feel advantages and disadvantages of science and technology which might produce great harm while benefiting human beings. Therefore, it is required to first integrate humanistic spirit and scientific spirit, achieve the combination of the true, the good and the beautiful, integrate rationality and emotion and realize harmonious unification of human and nature in modern scientific education. HPS education just complies with such requirements. It is the combination of three totally different disciplines. The philosophy of science and scientific sociology provide assistance and guidance for scientific education. Meanwhile, the history of science provides it with rich nutrient resources. It can be regarded as the result of perfect combination between humanistic spirit education and scientific education. Therefore, it is required to investigate mathematics in the wide social and cultural environment and integrate liberal arts and science during college mathematics teaching based on HPS teaching. Only when students have more rational understandings of mathematical science view and value can mathematical science promote social progress. This is the ultimate goal and requirement of college mathematics teaching.

\section{Urgent needs of students majoring in college mathematics}

Currently, science and technology have closer connection with our life, society and even culture. An essential quality of modern people is scientific literacy. The requirement of HPS education for scientific education is to train more scientific and technological elites and meanwhile improve scientific literacy of each citizen and allow educatees to have positive value and spirit of scientific exploration. Therefore, the application of HPS education to college mathematics education can allow mathematical scientific literacy to achieve greater progress, train more excellent mathematical talents and achieve greater development and progress of mathematics. Meanwhile, most college students have complete knowledge structure and thought and mature abilities of abstract thinking and philosophical thinking. Therefore, they can understand relevant contents of HPS education more easily. The application of HPS education in the stage of college has ideal effect and is an inevitable requirement for the development of college students.

\section{Specific strategies and main ways for applying HPS education to college mathematics teaching}

Mathematics teaching based on HPS education in colleges and universities is a strongly systematic project. Its educational concept and form are discussed and meanwhile its realization approach and strategies are explored. Though HPS education has multiple means and rich approaches and involves internal and external factors and dominant and recessive factors, the most common and main teaching approach of HPS education is effective classroom teaching process. Therefore, when teaching a new course and explaining exercises, college mathematics teachers should be good at grasping the opportunity of HPS education, combining mathematical professional knowledge and many other disciplines and contents such as history of mathematical science and social value, and reflecting the value and significance of college mathematics in practice fully, timely and accurately. To some extent, the effective application of HPS education in the teaching of college mathematics depends on effective HPS education in the classroom teaching process of mathematics.

\section{Select historical data and apply them in class}

HPS education emphasizes that the combined action of scientific sociology, history of science and philosophy of science jointly elaborates the essence of science and improves educational quality. The history of science studies the occurrence and development process of science. Due to the uniqueness of research perspective, history of science is a precious educational resource in scientific education. George Sutton was the founder of history of science. He believed that history of science connected natural science and humanistic studies and students could obtain the image of natural science and have comprehensive understandings of science and the relationship between science and humanity. Lanngevin Paul was a famous educator emerging at the beginning of the 20th century in France. He 
considered French scientific education failed because it only imparted dogmatic scientific knowledge to students and overstressed pragmatism. In the opinion of Lanngevin Paul, this method was a dull and boring repetitive process and turned science into a rigid and boring thing. The history of science proves that scientific knowledge is developing constantly. Therefore, the significance of history of mathematics is also very important for mathematical education. Students can realize how mathematicians conducted explorations in those days through study, thus having deeper understanding of the essence of mathematical science and mastering more methods of mathematical study.

According to features of HPS education, attention should be paid to the following during the selection of historical data. First, we should select historical data that can reflect the source and value of mathematical knowledge in the real life and help students develop correct concepts. Mathematical history can allow students to feel the important role of mathematics in the process of human cultural development and scientific progress and thus understand that mathematics is not simply symbols and formulas but also the most basic cultural activity of human beings, have correct understandings of the status of mathematics in social development and the relationship between mathematical science and other disciplines. For example, the discovery of Neptune is due to the application of mathematics in astronomy; American military solved a linear programming with several millions of variables in Gulf War and completed scheduling for the huge military and logistics system perfectly.

Second, we should select historical data that comprehensively show the whole process of problems of mathematical science ranging from their introduction to solving and subsequent development. Such historical data can train students' thinking on mathematical science with correct thoughts. Most teaching materials related to mathematics are prefect and correct. However, in other words, knowledge in such teaching materials is inserted into a standard template and restricts the freedom of students' way of thinking. Therefore, to integrate mathematical history into the classroom teaching of mathematics, we cannot simply impart knowledge to students. Instead, it is required to create an atmosphere for students to conduct explorations, discuss and understand an issue or concept positively and actively and master its development process. For example, "calculus" did not have the definition of "infinitesimal" as rigorous as it is now in the initial stage of creation. It was improved and became mature through constant efforts of later generations of mathematicians. By learning mathematical history, students can understand that mathematical problem is a process and the process from its generation to final solving is an active thinking process.

Finally, historical data selected should reflect personality charm and lofty sentiments of mathematicians and scientists. Under their influence, students' attitude and emotion towards mathematics will be improved. As any other science, mathematics also has an uneven development path. A lot of mathematicians have adhered to the truth and made great efforts, thus achieving the current glory. College students will be greatly inspired under the edification of such excellent qualities. For example, Archimedes was conducting research at the supreme moment of invasion of the enemy in order not to leave any theorem that he didn't prove. Even with blindness of the right eye, Euler did not give up mathematical study. Such history tells us that mathematics is not simply a tool and it also has rich humanistic connotations.

\section{The essence of HPS education is the integration, exchange and supplement of scientific sociology, philosophy of science and history of science}

In college mathematics teaching, attention should be paid to combine mathematics with students' real social life and make mathematical science and the society produce positive interaction. Famous Chinese mathematician Loo-Keng Hua thought that mathematics should be used for everything ranging from the universe to particles and even the change of earth. People can calculate the orbital velocity of curvilinear motion and Divine Series can lift off smoothly just as Newton found calculus through continuous efforts. Therefore, college mathematics teachers should make students feel that mathematics closely concerns social progress and human development in classroom teaching.

First, mathematical knowledge should be abstracted in combination with real life. Mathematical knowledge is study on quantitative relations in the objective world. Not everything has an original 
shape of mathematics. Therefore, teachers should have adequate observation ability and understanding, extract mathematical knowledge in life, transfer mathematical thought in combination with life phenomena and finally encourage students to fully apply the knowledge learnt in life and make the mathematical knowledge learnt more useful. Classroom teaching is not enough. Therefore, teachers should create conditions positively, e.g. lead students to study relevant social issues, and make students see the value of mathematics for actual life and be more enthusiastic about learning.

\section{Value of application of HPS education in college mathematics teaching}

\section{Contribute to the learning of mathematical knowledge and professional skills}

HPS education emphasizes the reappearance of history and process in science education. Different from direct impartation of mathematical knowledge in traditional mathematical science education, HPS education emphasizes the manifestation of development process of concepts and law and shows the richness, contingency and authenticity of the filtered history. Though traditional methods have higher efficiency of knowledge communication in the short time, students cannot feel the discovery and creation of mathematics in person. Mathematics teaching with HPS education in colleges and universities can lead students to improve their scientific knowledge and professional skills better.

\section{Stimulate students' enthusiasm about innovation}

Students are trained into receivers of knowledge under the traditional exam-oriented education mode. They do not discover new knowledge and methods actively. Many students with "high mark and mental deficiency" have been trained. HPS education emphasizes the combination of philosophy and history and great stimulation of students' innovation consciousness and enthusiasm about creation. A lot of scientific practice shows that scientific creation and development can be easily conducted under the combined action of history of science and scientific philosophy. For example, Einstein said that he created special relativity under the inspiration of a work on philosophy.

\section{Contribute to the training of students' sense of social repsonsibility}

HPS education not only imparts scientific knowledge and scientific research achievements to students, but also tells them due scientific attitude and trains their scientific literacy. Students start with aspects closely related to our life and society to learn scientific issues and pay adequate attention to issues concerning future social development such as environment, ecology and energy. Only after understanding the relationship between social life and science and technology and their functions can students know how to solve problems with science. Only with deeper understanding of connotations of mathematical science can students realize humanistic value and care implied. It can greatly improve students' sense of social responsibility and allow them to apply mathematical science to promote development progress of human beings and the society.

\section{Acknowledgments}

The topic name of this paper is Experimental Study on Application of Video Case Analysis Technology in Teacher Training; topic number: CJF07052. It is also a project of school educational reform named Study on Development Mode of Ability of Mathematical Teachers in Elementary and Secondary Schools to Solve Problems of Classroom Teaching.

\section{References}

[1] Sun Qingkuo, Hu Qizhou. View on Science Curriculum and Teaching Reform in China from Perspective of HPS. Journal of Jiangxi Institute of Education, 2013,34(3):16-19. 
[2] Li Dingping, Zhu Jianghua. Existing Problems of Mathematics Course Teaching Reform in Colleges and Universities and Countermeasures. Curriculum and Educational Studies, 2015,(6):38-39.

[3] Wang Qian. Analysis on Mathematics Education in Colleges and Universities from Perspective of Mathematical Culture. Curriculum and Educational Studies, 2015,(5):137-137,138.

[4] Wang Yaling. Exploration and Practice of Mathematics and Applied Mathematics Major Construction in Colleges and Universities. Archive, 2014,(12):195.

[5] Xu Jiang. Inspiration of International HPS Education and Scientific Teaching on Scientific Educational Reform in China. Journal of Hunan University of Science and Technology (Social Sciences Edition), 2011,14(6):175-179. 